\title{
Spatially Resolved Study of Backscattering in the Quantum Spin Hall State
}

\author{
Markus König, ${ }^{1,2}$ Matthias Baenninger, ${ }^{1,2}$ Andrei G. F. Garcia, ${ }^{1}$ Nahid Harjee, ${ }^{3}$ Beth L. Pruitt, ${ }^{4}$ C. Ames, ${ }^{5}$ \\ Philipp Leubner, ${ }^{5}$ Christoph Brüne, ${ }^{5}$ Hartmut Buhmann, ${ }^{5}$ Laurens W. Molenkamp, ${ }^{5}$ and David Goldhaber-Gordon ${ }^{1,2, *}$ \\ ${ }^{1}$ Department of Physics, Stanford University, Stanford, California 94305, USA \\ ${ }^{2}$ Stanford Institute for Materials and Energy Sciences, SLAC National Accelerator Laboratory, \\ 2575 Sand Hill Road, Menlo Park, California 94025, USA \\ ${ }^{3}$ Department of Electrical Engineering, Stanford University, Stanford, California 94305, USA \\ ${ }^{4}$ Department of Mechanical Engineering, Stanford University, Stanford, California 94305, USA \\ ${ }^{5}$ Physikalisches Institut (EP3), Universität Würzburg, Am Hubland, 97074 Würzburg, Germany
} (Received 16 November 2012; published 22 April 2013)

\begin{abstract}
The discovery of the quantum spin Hall (QSH) state, and topological insulators in general, has sparked strong experimental efforts. Transport studies of the quantum spin Hall state have confirmed the presence of edge states, showed ballistic edge transport in micron-sized samples, and demonstrated the spin polarization of the helical edge states. While these experiments have confirmed the broad theoretical model, the properties of the QSH edge states have not yet been investigated on a local scale. Using scanning gate microscopy to perturb the QSH edge states on a submicron scale, we identify well-localized scattering sites which likely limit the expected nondissipative transport in the helical edge channels. In the micron-sized regions between the scattering sites, the edge states appear to propagate unperturbed, as expected for an ideal QSH system, and are found to be robust against weak induced potential fluctuations.
\end{abstract}

DOI: 10.1103/PhysRevX.3.021003

Subject Areas: Mesoscopics, Topological Insulators

\section{INTRODUCTION}

Ever since the prediction and realization of topological insulators, this new class of material has not only been studied for fundamental scientific reasons but has also garnered significant interest because of potential applications. Specifically, the quantum spin Hall (QSH) edge channels appear to be a promising candidate for low-power signal transmission, as they are expected to exhibit dissipationless transport. In the quantum spin Hall state [1,2], backscattering between the counterpropagating channels in one helical edge state is expected to be suppressed by perfect destructive interference as long as the Fermi level is located in the bulk gap, time-reversal symmetry is preserved, and interactions are weak [3,4]. In HgTe quantumwell structures, the QSH state was predicted to exist if the thickness of the quantum-well layer exceeds a critical value of $d_{\mathrm{QW}} \approx 6.3 \mathrm{~nm}[5]$. The bulk energy gap in this material is of order $10 \mathrm{meV}$, so room-temperature applications would require other materials such as thin films of $\mathrm{Bi}_{2} \mathrm{Te}_{3}$ [6]. However, HgTe can serve as a model system for the investigation of the QSH state: Following the initial observation of the QSH state in HgTe [7], the edge-state nature of transport [8] and the spin polarization of the edge states [9] are experimentally demonstrated in the same material. The good agreement between the experimental

\footnotetext{
*goldhaber-gordon@stanford.edu
}

Published by the American Physical Society under the terms of the Creative Commons Attribution 3.0 License. Further distribution of this work must maintain attribution to the author(s) and the published article's title, journal citation, and DOI. results and theoretical predictions based on the LandauerBüttiker formalism confirms that transport in the helical edge channels is indeed ballistic over short distances. However, conductance values indicative of ballistic transport are only observed on edges shorter than a few microns [7,8,10]. In Ref. [8], a measured nonlocal conductance value could be explained within the Landauer-Büttiker formalism, assuming a single scattering site that fully equilibrates the counterpropagating channels along one particular segment of the device edge. The equilibration is attributed to dephasing of the helical states in a metallic region coupled to the edge states. Such a metallic puddle could form in an inhomogeneous potential landscape introduced by sample growth or processing. In multiterminal devices, the metallic contacts to the QSH edge states can be treated as macroscopic regions causing equilibration, explaining the experimentally observed quantized resistance of the QSH state $[7,8,10]$. Besides dephasing in metallic regions, various other ways to induce backscattering in a helical edge state have been studied theoretically, including mechanisms based on magnetic [11] or nonmagnetic [12] impurities, spin-orbit interaction [13,14], or phonons [15]. Against this background of theoretical effort to investigate scattering mechanisms in the QSH state and the obvious presence of backscattering in transport measurements, more detailed experimental investigations of scattering are urgently needed.

In this paper, we use scanning gate microscopy to locally manipulate the QSH edge states in HgTe structures. In the next section, we will introduce the experimental approach in more detail and will also specify the properties of the studied samples. Subsequently, we will present 
experimental results obtained by scanning gate microscopy on a device in the QSH regime where signatures indicative of locally induced backscattering of the edge states can be found. These results are complemented by measurements in a transport regime characterized by the coexistence of bulk and edge states. The behavior observed in both regimes will be discussed based on the presence of small metallic regions that lead to dephasing of the QSH edge states.

\section{EXPERIMENTAL METHODS}

A promising approach to study backscattering in the helical edge states on a local scale is to generate small local potential fluctuations and test whether they perturb transport in the edge channels. To achieve this goal, one must induce a local potential perturbation with precise control over its position and strength. This approach allows either modulation of the strength of a preexisting scattering site or the generation of an artificial scattering site. In scanning gate microscopy (SGM) [16,17], a charged tip is scanned above the device of interest, and the effect of the induced potential fluctuation on the conductance in the device is monitored. Over the past few years, scanning gate microscopy has been used to investigate transport phenomena in a variety of low-dimensional systems like quantum dots [18-21], quantum-point contacts [22], and two-dimensional electron gases [23-26]. Of particular relevance to QSH edge-state transport, SGM has been applied to one-dimensional systems like carbon nanotubes $[27,28]$ and nanowires [29], where it was used to identify and manipulate localized states that control the transport through the device.

Five devices were fabricated from two undoped $\mathrm{HgTe} / \mathrm{Hg}_{0.3} \mathrm{Cd}_{0.7} \mathrm{Te}$ quantum-well structures with nominal HgTe layer thicknesses $d_{\mathrm{QW}}$ of 7 and $8 \mathrm{~nm}$, respectively. The devices were patterned using optical lithography, ion milling to define mesas, and evaporation of In/Au ohmic contacts to the quantum well. Figure 1(a) shows a section of a typical device in a topographic image taken by atomic force microscopy. Our home-built SGM probes feature self-sensing piezoresistive deflection readout [30], which allows for precise in situ alignment of the tip to the device. The separation between the contacts in the transport direction is $5 \mu \mathrm{m}$, and the lateral mesa width is $150 \mu \mathrm{m}$. As this width is several orders of magnitude greater than the predicted extension of the QSH edge states into the bulk [31,32], any effect of interedge tunneling [33-35] can be ruled out. More relevant to our SGM experiments, the large width of the device ensures that the tip-induced potential perturbation only affects the transport along the edge located within the scan window, while the far edge remains unaffected and thus provides a constant contribution to the device conductance. We perform our experiments in a twoterminal configuration using a standard lock-in technique $\left(f \approx 830 \mathrm{~Hz}, U_{\mathrm{exc}}=100 \mu \mathrm{V}\right)$ at $T \approx 2.7 \mathrm{~K}$. Despite the
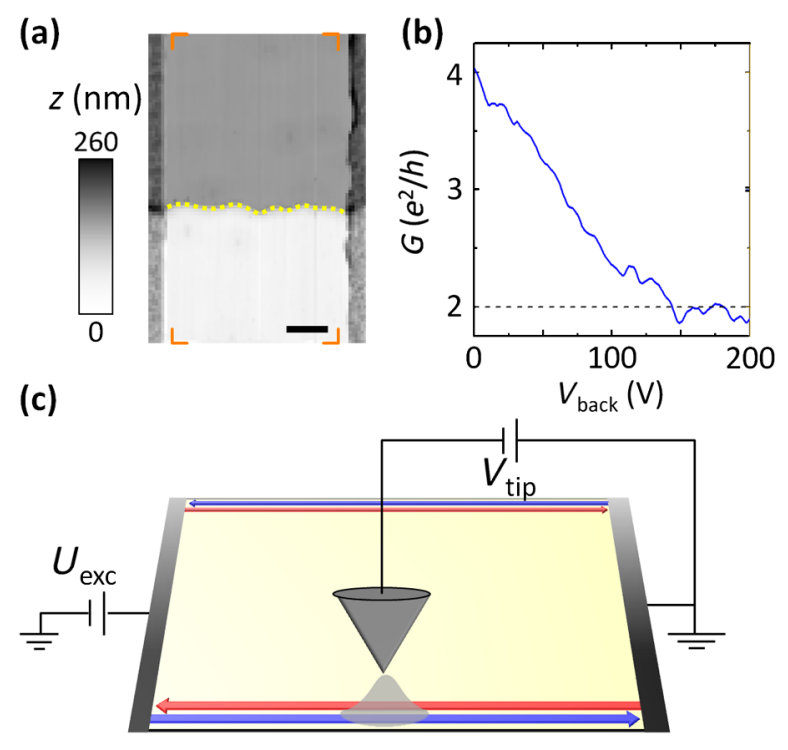

FIG. 1. (a) In situ atomic force microscopy scans (scale bar $1 \mu \mathrm{m}$ ) allow for precise alignment of the tip to the device. The mesa extends upward from the dotted yellow line. The grainy areas at the left and right edges, respectively, are the Ohmic contacts to the quantum well. The orange markers demarcate the scan window used for the conductance maps in Figs. 2 and 3. (b) A back-gate electrode is used to tune the device into the QSH regime with $G \leq 2 e^{2} / h$. (c) Illustration of the experimental configuration for SGM studies of the QSH state.

absence of intentional doping in the heterostructure, the studied quantum-well structures consistently show a finite density of $p$-type bulk carriers, typically on the order of a few times $10^{10} \mathrm{~cm}^{-2}$. For the device discussed extensively in this paper, we find a density $p \approx 2 \times 10^{10} \mathrm{~cm}^{-2}$, prior to gating [36]. Figure 1(b) shows the device conductance as a function of the voltage applied to the back-gate electrode, which is used to tune the Fermi level in the device [37]. The observed saturation of the conductance slightly below $G=$ $2 e^{2} / h$ for $V_{\text {back }}>150 \mathrm{~V}$ suggests that the sample is in the QSH state and that transport happens only along the edges. The basic concept of SGM measurements on a sample in the QSH state is illustrated in Fig. 1(c): A dc voltage $V_{\text {tip }}$ is applied to the tip located slightly above the sample surface. (In our experiments, height $h=90 \mathrm{~nm}$.) The electric field from the tip produces a local modulation of the potential in the sample, below the tip. In our SGM experiments, we induce such a potential perturbation near the device edge with the goal of introducing and controlling backscattering of the helical edge channels. All devices show comparable results (see the Supplemental Material [36]), except as noted. In this paper, we focus our discussion on one particular device $\left(d_{\mathrm{QW}}=8 \mathrm{~nm}\right)$, which shows the same qualitative features as other devices as well as striking and enlightening behavior seen only on this device. While the data do not allow for a fully conclusive study of scattering mechanisms that affect the QSH edge channels, our results 
nonetheless provide a substantial step forward over the available transport experiments.

\section{RESULTS AND DISCUSSION}

\section{A. Scanning gate microscopy in the QSH regime}

In the QSH regime at $V_{\text {back }}=+200 \mathrm{~V}$, we observe a strong modulation of the conductance in the SGM maps on this device [Figs. 2(a)-2(d)]: As a function of the tip position, the conductance varies approximately from $2 e^{2} / h$ (similar to the conductance value when the unbiased tip is far away) down to $1 e^{2} / h$. The rather smooth variation of $G$ over a length scale of several microns in the tip position perpendicular to the edge of the device, most clearly visible in Fig. 2(a), is mostly caused not by a local gating by the tip but rather by long-range gating originating from both the tip and the large conductive area of the cantilever biased to the same voltage as the tip. In particular, for a strongly negative tip voltage $V_{\text {tip }}=-14.5 \mathrm{~V}$ [Fig. 2(a)], regions with $G>2 e^{2} / h$ can be observed in the conductance map. Such a conductance value cannot be explained solely by transport in the QSH edge states, and we attribute the excess conductance to the tip-induced emergence of bulk conductance (see the Supplemental Material [36]). In contrast, the superimposed modulations on length scales below $1 \mu \mathrm{m}$, highlighted by taking the gradient of conductance with tip position in Figs. 2(e)-2(h), are associated with local gating by the SGM tip, showing the effect of a well-localized potential perturbation on the edge-state conductance. The suppression of conductance by $|\Delta G| \approx 1 e^{2} / h$ by local gating suggests that transport at one of the two edges is fully suppressed by the tip potential. This result supports our assumption that the sample is in the QSH state, with transport occurring primarily along the edges of the device.
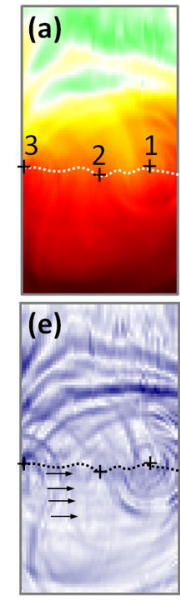
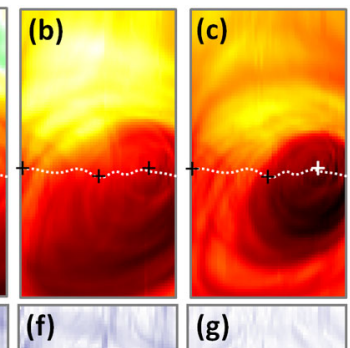

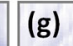

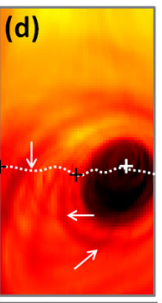

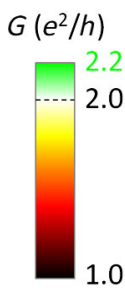

(h)

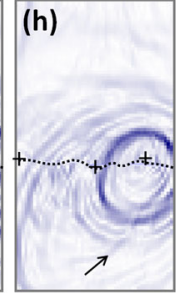

$|\nabla G|$ (arb.u.)

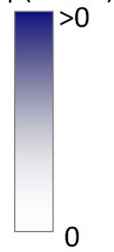

FIG. 2. (a)-(d) Conductance maps $G(x, y)$ with $V_{\text {back }}=$ $+200 \mathrm{~V}$ for $V_{\text {tip }}=$ (a) -14.5 , (b) -12.5 , (c) -11.0 , and (d) $-9.5 \mathrm{~V}$. The dotted lines indicate the edge of the device. The labeled crosses mark the positions of the scattering sites. (e)-(h) Gradient $|\nabla G(x, y)|$ of (a)-(d).

As noted above, some prior transport measurements [8] may be understood by invoking a single fully edgeequilibrating metallic region along a single edge. Full equilibration would decrease the conductance of that edge by a factor of 2 , from $1 e^{2} / h$ to $0.5 e^{2} / h$. Based on a simple cartoon picture for our SGM experiments, we expected the tip potential to induce such a local metallic region below the tip, so we are surprised to see a stronger suppression than $0.5 e^{2} / h$ associated with a single site. We find only a single occurrence of full suppression of edgestate transport in all of the measured devices, so our data do not allow for a conclusive identification of an underlying mechanism for the observed full suppression of edge-state transport. Nonetheless, as such behavior is intriguing and may be interesting for the reader, in the Supplemental Material [36], we discuss the applicability of several theoretical models that could in principle lead to the full suppression of edge conduction [11,13,38-40]. For the remainder of the paper, we focus on the more commonly seen features in the SGM scans-circular features in the SGM maps indicative of resonant backscattering. Comparable features are observed in all other devices as well [36].

\section{B. Resonant backscattering of QSH states}

Besides the pronounced conductance suppression attributed to a single site, the conductance maps show multiple sets of concentric rings that represent a reduced conductance. Around site 1, the rings are superimposed on the strong conductance modulation (the full suppression). Two more sets of concentric rings are centered around sites 2 and 3, respectively, in Fig. 2. While these features are partly masked in the conductance maps by the dominant conductance modulation originating from site 1 , they become more visible when the gradient of the conductance is plotted: Features about site 3 can be seen in all gradient maps [Figs. 2(e)-2(h)]. While it is difficult to identify complete rings around site 2 , segments of several rings are visible in Figs. 2(d) and 2(h) (highlighted by arrows). For all three scattering sites, the conductance modulation associated with the sharp rings of conductance suppression is on order $0.1 e^{2} / h$. Circular patterns with a similar conductance modulation are found in all the devices we study.

In general, circular patterns in SGM maps are a signature that transport is a function of the potential at one sensitive site, the common center of the circles. The circular shape can be understood intuitively: When the tip moves along one of the circles, the tip-induced potential at the circle's center does not change and the conductance remains unaffected. However, when the tip moves toward or away from the sensitive site, the potential at that site and, consequently, the conductance change. The canonical example of this behavior is a quantum dot, where the local potential at the dot determines the occupancy, and the conductance peaks in Coulomb-blockaded transport show 
up as rings in SGM experiments [19,28,29]. The observation of ringlike patterns in our data suggests that the suppression of conductance in the QSH edge channel is linked to individual sites located at or near the physical edge of the device, although, in place of the Coulomb blockade, we suggest below another mechanism dependent on the local potential. In our experiments, the rings of conductance suppression appear elongated because of spatial variation in the dielectric environment: When the tip is located above the mesa, the tip potential experienced at the scattering site is screened by the $\mathrm{Hg}_{0.3} \mathrm{Cd}_{0.7} \mathrm{Te}$ cap layer in the heterostructure with $\epsilon_{\mathrm{HgCdTe}}=12.7$, whereas, for a tip position not above the mesa, the relevant dielectric constant should be between $\epsilon_{\mathrm{HgCdTe}}$ and $\epsilon_{\mathrm{vacuum}}=1$.

The magnitude and other experimental signatures of conductance modulation that result in multiple rings about the three indicated scattering sites may be understood if these sites are associated with small bulk conducting regions that adjoin the edge and serve as sources of dephasing [8]. For simplicity, we will discuss transport only through the helical edge state we scan over and omit the contribution of $G=1 e^{2} / h$ by the far edge. A macroscopic metallic contact that is well coupled to the edge is expected to fully equilibrate the counterpropagating edge states as noted above. As there is no relationship between the phase of an electron impinging on this contact and one emerging back into the edge, backscattering paths interfere randomly rather than perfectly destructively, and the electron should emerge with equal probability into the forward-moving and backward-moving channels, respectively. Consequently, conductance along the edge should be reduced from $G=$ $1 e^{2} / h$ to $G=0.5 e^{2} / h$. A metallic region of intermediate size could also reduce the edge conductance: If the phase of impinging electrons is partly randomized through interaction with charge carriers in the metallic puddle, backscattering paths no longer perfectly destructively interfere, so backscattering is possible. In Ref. [8], "dephasing" in the puddle was introduced phenomenologically to the model rather than quantitatively derived from a particular mechanism; however, electron-electron interaction or electron-phonon interaction were suggested as possible sources for this dephasing. The amount of conductance decrease would depend on the exact size of the dephasing region and the strength of the dephasing process. In particular, the finite size of the metallic puddle should produce discrete energy levels. When the metallic region's size is such that the Fermi level is aligned with one of those discrete levels, electrons can enter the metallic region easily and experience dephasing, leading to strong suppression of edge-state conductance. This resonant condition should hold for particular sizes of the metallic region. The conductance suppression caused by this resonant process is predicted to be around $0.1 e^{2} / h$ [8], comparable to the size of the modulation observed in our SGM maps. In the experimental data accompanying the model put forward in Ref. [8], however, similar resonances were not observed, pointing toward the presence of a metallic puddle that results in strong dephasing of the QSH edge state, e.g., because of a very large size.

Our experimental results indicate that backscattering of the QSH edge state is caused by well-localized scattering sites along the edge of the device. The observed pattern in the conductance modulation is consistent with the theoretical model described above that is based on partial dephasing of the edge state in a small metallic region at each of these sites. Subsequently, we will use this picture to interpret the backscattering observed in our measurements. When either the tip position or the tip voltage is changed, the potential at the puddle will vary, modulating the puddle size and possibly the strength of the dephasing mechanism. This modulation should tune the dephasing process in the metallic region in and out of resonance, resulting in an oscillatory conductance modulation. Electrostatic simulations show that the size of a metallic region-either induced by the tip potential itself or already existing in the sample - with a charge density exceeding a certain threshold value can be tuned by a few hundred nm within the tipvoltage range used in our experiments [36]. At the same time, the maximum density in the puddle, which should affect the strength of the dephasing mechanism, changes with the tip voltage as well. This variation in the size and strength of the dephasing region is expected to result in multiple resonances [8], as seen in our experimental data.

Our explanation for the observed backscattering is corroborated by additional features that appear at strongly negative tip voltages $V_{\text {tip }} \leq-12.5 \mathrm{~V}$. The maps of $|\nabla G|$ show multiple lines that run approximately parallel to the mesa edge along the entire length of the device [indicated by arrows in Fig. 2(e) and Fig. S7 in the Supplemental Material [36] ]. This pattern of the gradient corresponds to an oscillatory conductance modulation as a function of the tip position perpendicular to the mesa edge. We can rule out simple instrumental artifacts as follows: The SGM data are recorded during line scans in the direction of the observed conductance modulation, so a slow drift in the sample conductance can be ruled out as an explanation. Instrumental oscillations on a time scale comparable to the measurement time between individual lines cannot account for the observed pattern either, as the lines only appear near the edge, evolve consistently with the applied tip voltage [36], and only occur for particularly large tip voltages. Although the amplitude of this conductance modulation is less than $0.05 e^{2} / h$-weaker than the effect caused by the localized scattering sites-it can be explained by the same mechanism. For a sufficiently strong potential perturbation induced by the SGM tip, a metallic puddle (here $p$ type because of the negative tip voltages) is induced at an otherwise unperturbed section of the edge. For a dephasing region of appropriate size, the resonant suppression of conductance should be seen. These lines are only visible 
for much stronger tip voltages $V_{\text {tip }} \leq-12.5 \mathrm{~V}$ compared to the ring features described above. This difference in tip voltage required to cause backscattering can provide a measure for the strength of the intrinsic potential fluctuations and the robustness of the otherwise unperturbed edge states, as discussed in more detail below. While the SGM maps for the device in the QSH regime provide clear evidence for the presence of scattering sites, experimental data for this transport regime are only available for a limited tip-voltage range $-14.5 \mathrm{~V} \leq \mathrm{V}_{\text {tip }} \leq-9.5 \mathrm{~V}$, as an accidental tip crash damaged the device at this point. However, earlier measurements on the same device in the presence of bulk carriers complement the data and allow for a detailed analysis; so, we move next to the discussion of the results obtained in the regime of mixed bulk and edge conduction. We will show later that we can analyze the resonant backscattering of the QSH edge states in small metallic regions despite the presence of bulk carriers.

\section{Coexistence of edge and bulk conductance}

At $V_{\text {back }}=0$, the sample displays a conductance $G \approx$ $4 e^{2} / h$. The conductance value larger than $2 e^{2} / h$ indicates that the device must have some bulk conductance. However, we expect QSH edge states to coexist with bulk carriers and contribute considerably to the total conductance. In this regime, the strongest local gating effect close to the edge reduces the conductance by $0.5 e^{2} / h$ [Figs. 3 and 4(a)]. As this conductance modulation can be induced within a few hundred nm of the edge, it is likely associated with an edge state and constitutes a lower limit for the conductance of a 5- $\mu \mathrm{m}$ stretch of a single edge state under these slightly $p$-type conditions. A possible and consistent scenario is that edge conductance is reduced in comparison to the QSH regime, possibly because coexisting bulk carriers allow backscattering of the edge states in the regions between the local scattering sites that were identified earlier. The reduced edge-state conductance compared to the QSH regime at $V_{\text {back }}=+200 \mathrm{~V}$ further confirms that the conductance along the edge is indeed because of QSH edge states and not caused by trivial edge currents, as they may occur because of an inhomogeneous potential landscape. At $V_{\text {tip }}=-3.5 \mathrm{~V}$, the strongest conductance modulation $|\Delta G| \approx 0.5 e^{2} / h$ is only achieved when the tip is located directly above the scattering site. This observation means that the potential perturbation at site 1 required for causing this suppression is equal to the maximum tip-induced perturbation for $V_{\text {tip }}=-3.5 \mathrm{~V}$. If the tip is moved away from site 1 , the induced potential is no longer sufficient to cause the strong suppression. Thus, the range of tip locations over which the conductance suppression can be observed- $125 \mathrm{~nm}$ (half width at half maximum) - can give an estimate for the size of the dephasing region [Fig. 4(a)]. The identification of this feature as the suppression of edge-state conductance is supported by the observation of an associated ring pattern centered at the edge of the mesa [Fig. 3(a)-3(c)], similar to those observed in the QSH regime. We can see two more sets of rings, and all three sets are centered around the same three locations as in the QSH regime and presumably to the same scattering sites. Thus, we can use these data for a more complete analysis of the properties of the dephasing regions. While the suppression caused by site 1 is unusually strong and the underlying mechanism is not understood yet, the superimposed resonances are qualitatively comparable to the features centered at scattering sites 2 and 3. As the conductance modulation associated with site 1 is most clearly visible in the SGM maps, we use scattering site 1 for further analysis.

Figure 4(b) shows the conductance measured along the line marked in Fig. 3(a) as a function of $V_{\text {tip }}$. We observe the resonant suppression of conductance corresponding to rings in the SGM maps, mainly for negative tip voltages. As the tip voltage becomes more negative, the spatial separation between the conductance dips increases. This shift can be understood based on the electrostatics of the SGM configuration. When the tip voltage is changed, the
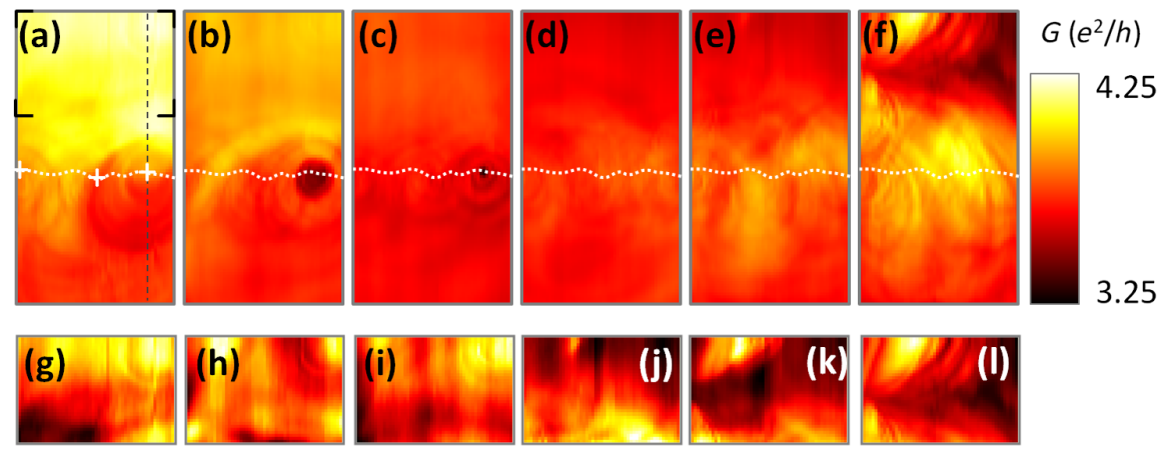

FIG. 3. (a)-(f) Conductance maps $G(x, y)$ taken at $V_{\text {back }}=0$ with tip voltages $V_{\text {tip }}=$ (a) -10.0 , (b) -6.5 , (c) -3.5 , (d) +3.5 , (e) +6.5 , and (f) $+10.0 \mathrm{~V}$, respectively. The vertical line in (a) indicates the position of the line scans shown in Fig. 4. (g)-(l) Transport signatures of localized states in the bulk. [The window is indicated in (a); the color scale $(\Delta G)$ is individually saturated for each scan.] (g) $V_{\text {tip }}=-10.0 \mathrm{~V}$ and $\Delta G=0.25 e^{2} / h$. (h) $V_{\text {tip }}=-8.0 \mathrm{~V}$ and $\Delta G=0.15 e^{2} / h$. (i) $V_{\text {tip }}=-6.5 \mathrm{~V}$ and $\Delta G=0.15 e^{2} / h$. (j) $V_{\text {tip }}=+6.5 \mathrm{~V}$ and $\Delta G=0.20 e^{2} / h$. (k) $V_{\text {tip }}=+8.0 \mathrm{~V}$ and $\Delta G=0.40 e^{2} / h$. (l) $V_{\text {tip }}=+10.0 \mathrm{~V}$ and $\Delta G=0.85 e^{2} / h$. 

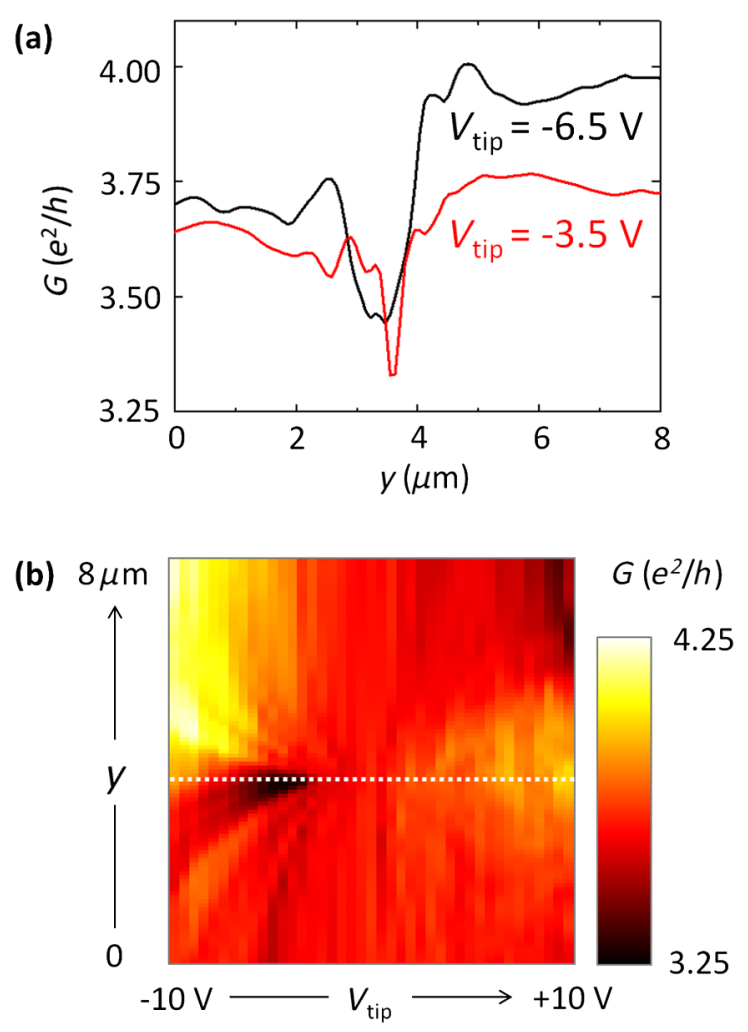

FIG. 4. (a) Line scans [line indicated in Fig. 3(a)] for $V_{\text {tip }}=$ $-6.5 \mathrm{~V}$ (upper black trace) and $V_{\text {tip }}=-3.5 \mathrm{~V}$ (lower red trace) show the strongest conductance modulation $\Delta G \approx 0.5 e^{2} / h$ and the most localized gating effect $\Delta y \approx 0.125 \mu \mathrm{m}$ (HWHM), respectively. (b) Evolution of line scans as a function of $V_{\text {tip }}$.

potential at a particular sensitive site within the sample can be kept constant if the distance between tip and site is adjusted accordingly, as traced out by the bright and dark curves in Fig. 4(b). More precisely, the tip voltage needed to trigger dephasing is more negative the farther away from the site the tip is, showing that the potential at the site needs to be pushed up for dephasing. While the strong local gating effect with $|\Delta G| \approx 0.5 e^{2} / h$ can only be observed for $V_{\text {tip }} \leq-3.5 \mathrm{~V}$, weaker resonances with $|\Delta G|<$ $0.1 e^{2} / h$ can still be observed for smaller negative tip voltages. In the slightly $p$-conducting regime at $V_{\text {back }}=0$, backscattering at the three identified scattering sites occurs for tip voltages as small as $V_{\text {tip }} \approx-1.0 \mathrm{~V}$ (sites 1 and 2) or $V_{\text {tip }} \approx-2.5 \mathrm{~V}$ (site 3 ), whereas detectable backscattering cannot be induced in the adjacent regions for tip voltages as strong as $-10 \mathrm{~V}$. This difference in tip voltage shows that the potential at the sensitive sites needs to be pushed up less to trigger dephasing than would be needed in the surrounding regions.

While there might be quantitative differences between backscattering in the QSH regime $\left(V_{\text {back }}=+200 \mathrm{~V}\right)$ and in the presence of weak bulk transport $\left(V_{\text {back }}=0\right)$, respectively, we will use $V_{\text {tip }} \approx-12.5 \mathrm{~V}$, which is necessary in the QSH regime to introduce backscattering in otherwise unperturbed regions of the edge, as an estimate for the strength of a potential perturbation required to cause backscattering. This tip voltage corresponds to a locally induced density $p \approx 2.5 \times 10^{11} \mathrm{~cm}^{-2}$ [36]. Let us assume that densities associated with backscattering at the identified sites are also around $p=2.5 \times 10^{11} \mathrm{~cm}^{-2}$. But, the low tip voltages that are necessary to turn on backscattering at those sites correspond to tip-induced density changes only on the order of several $10^{10} \mathrm{~cm}^{-2}$. This discrepancy between the respective carrier densities implies that the metallic puddles at the scattering sites are already present in the absence of the tip for $V_{\text {back }}=0$. In addition, tuning the sample into the QSH regime by applying $V_{\text {back }}=$ $+200 \mathrm{~V}$ changes the bulk density by $|\Delta p| \approx 2 \times$ $10^{10} \mathrm{~cm}^{-2}$, i.e., significantly less than the inferred carrier density in the dephasing regions, indicating that the resonant backscattering observed in the QSH regime at $V_{\text {back }}=$ $+200 \mathrm{~V}$ can be attributed to $p$-type puddles that interrupt the edge state. The much larger carrier density in the dephasing regions compared to the density induced by the back gate means that it is reasonable to study the backscattering of QSH edge states even in a range of back-gate voltage where bulk and edge transport coexist, and to qualitatively apply what we learn to the QSH regime as well. The comparatively high densities required for dephasing in a metallic puddle may also explain why bulk densities of a few $10^{10} \mathrm{~cm}^{-2}$ still allow for significant edge-state transport. Some of the present authors recently studied current flow patterns in $\mathrm{HgTe}$ devices by scanning SQUID magnetometry, reporting that a significant proportion of current flowed at edges up to even higher bulk densities than explored here [41]. This coexistence of edge and bulk transport suggests that edge-state transport is rather robust against the presence of bulk carriers. An explanation for the apparently weak interaction between edge and bulk states might be provided by the mismatch between the wave vectors of the edge and bulk states [42].

All observed scattering sites can be identified as $p$ type, whereas the experimental signatures of preexisting $n$-type puddles are absent. This disparity may be related to the band structure of the HgTe structures: Hole densities of a few $10^{11} \mathrm{~cm}^{-2}$ can already occur in the quantum well if the Fermi level is just $1 \mathrm{meV}$ below the valence band maximum, much less than the shift into the conduction band required to induce comparable $n$-type densities [36], so that potential fluctuations of a few meV might be responsible for the observed metallic scattering sites. As our measurements imply that densities on the order of $10^{11} \mathrm{~cm}^{-2}$ are required to cause substantial backscattering, a conductance $G \approx 0.5 e^{2} / h$ carried by a helical edge state that coexists with a bulk carrier density $p \approx$ $2 \times 10^{10} \mathrm{~cm}^{-2}$ at $V_{\text {back }}=0$, as determined earlier, appears reasonable.

For positive tip voltages, the pattern of conductance modulation in the SGM maps becomes significantly more 
complex [Figs. 3(d)-3(f)], so that the attribution of individual features to particular scattering sites is rarely possible. The clearest features at the mesa edge are centered around site 3, where circular patterns are visible for both positive and negative tip voltages, suggesting that the site is not simply the location of a preexisting potential fluctuation.

A variation of the well width by just a single monolayer can change the bulk 2D energy gap by several meV, which is significant compared to the $13-\mathrm{meV}$ gap predicted for our 8-nm quantum well [36]. These comparable energy scales could naturally lead to a small region where local gating could enable both $p$ - and $n$-type puddles at the same location. At the same time, a variation of the bulk gap could also result in a relative shift of the bands, locally shifting the Fermi level from the gap into one of the bulk bands.

\section{Nature of bulk transport at low densities}

When we position the tip over the slightly $p$-conducting bulk at $V_{\text {back }}=0$, we see an overall trend from high conductance $G$ for negative tip voltages to low conductance for positive tip voltages [Figs. 3(a)-3(f)]. The increase in $G$ for negative tip voltages [Figs. 3(a)-3(c)] can be understood as because of a tip-induced accumulation of $p$-type bulk carriers. For positive tip voltages [Figs. 3(d)-3(f)], the conductance is suppressed by approximately $0.5 e^{2} / \mathrm{h}$ compared to the conductance in the absence of a tip effect. Although the sign of this effect makes sense, its magnitude is several times stronger than one would expect for the full suppression of bulk transport through only a small fraction of the device-probably around a few percent of the device width - in the case of a homogeneously conducting bulk with $G_{\text {bulk }}<4 e^{2} / h$. But, this assumption of homogeneous conduction is not justified. If we assume a conductance $G \geq 0.5 e^{2} / h$ for each edge state in the slightly $p$-conducting device at $V_{\text {back }}=0$, as determined above, we obtain a bulk resistivity that exceeds a few hundred $\mathrm{k} \Omega / \square$, a range characteristic of percolative or hopping bulk transport. In this transport regime, signatures of localized states are visible in the SGM maps, not just along the edge of the device but also in the bulk of the sample.

These features are located several microns away from the edge [Figs. 3(g)-3(1)], significantly more than the approximately $100-\mathrm{nm}$ theoretical [31,32] and experimental [43] estimates for the width of QSH edge states. Thus, the observed behavior is likely representative of bulk transport in the low-density regime, despite some proximity to the device edge. In contrast to the superficially similar sets of rings centered at the edge, the localized states in the bulk manifest themselves in a variety of conductance patterns. For negative tip voltages [Figs. 3(g)-3(i)], for example, a set of concentric sharp rings with enhanced conductance is visible in the upper right corner of the scan window, marking resonantly enhanced transport, as is typical for a quantum dot [18-20,29]. In the scans with $V_{\text {tip }} \geq+6.5 \mathrm{~V}$, a single elongated region of enhanced conductance emerges at the upper edge of the scan window and grows with increasing tip voltage [Figs. 3(j)-3(l)]. Such a response to a locally induced potential is reminiscent of the behavior seen in SGM studies of quantum-point contacts (QPCs) where local gating can alter the transmission of a QPC, producing a significant change in conductance $[22,44]$. The enhancement of conductance with increasingly positive tip voltages implies that the tip induces $n$-type carriers. This scenario is very plausible, as the $p$-type densities extracted above for negative tip voltages are significantly larger than the $p$-type background density in the absence of the tip, and the small band gap of HgTe quantum wells allows for a tip-induced shift of the Fermi level from the valence band into the conduction band. For large positive tip voltages, a set of sharp arcs of suppressed conductance [Fig. 3(1)] could point to the presence of another localized state that strongly affects transport in our device. The center of these arcs appears to be in the bulk outside the scan window but is probably still inside the channel in which we measure conductance. All these observations are consistent with a two-dimensional system with strong potential disorder, in which quantum dots and quantum-point contacts can accidentally form as the Fermi level fluctuates between bands and gap.

In this context, we note that the SGM maps taken at $V_{\text {back }}=+200 \mathrm{~V}$ (Fig. 2) do not show any comparable signs of transport through localized states in the bulk. This lack of features away from the edge supports our interpretation that the device is in the QSH regime; i.e., the bulk is truly insulating for this back-gate voltage.

\section{CONCLUDING REMARKS}

The key results of our experiments are evidence of edge-state transport in the QSH regime, identification of preexisting scattering sites, and demonstration that backscattering of the QSH states caused by these sites can be enhanced resonantly if the local perturbation is appropriately tuned. The separation between these well-localized scattering sites is typically between 1.5 and $2 \mu \mathrm{m}$, and the edge states appear to propagate unperturbed between them. This spacing qualitatively explains the size limit of 1 to a few microns for ballistic transport in QSH devices determined in earlier transport experiments $[7,8,10]$ while also suggesting that, if scattering sites could be individually tuned off resonance, the ballistic length could be substantially extended. The detection of localized states in the bulk supports our interpretation that the scattering sites along the edge can be attributed to local disorder, which is inherent to the quantum-well structure and not a result of damage imposed on the edge of the device during the fabrication process. In addition, we have demonstrated that backscattering can be introduced in otherwise unperturbed QSH states by a sufficiently strong tip-induced 
perturbation. The purely tip-induced backscattering gives insight into the robustness (or vulnerability) of the QSH states against potential fluctuations. However, as the conductance modulation solely induced by the tip potential is weaker than the effect of the preexisting scattering sites identified in our current experiments, it would be worthwhile to study these effects in more detail in subsequent experiments. Similar SGM experiments in an external magnetic field could elucidate the role of time-reversal symmetry for the predicted suppression of backscattering.

\section{ACKNOWLEDGMENTS}

We thank M.R. Calvo, J. Maciejko, K. Moler, K. Nowack, and T. Schmidt for valuable discussions. This work was funded by the Department of Energy, Office of Basic Energy Sciences, Division of Materials Sciences and Engineering, under Contract No. DE-AC02-76SF00515 (SGM and transport investigation of the QSH state in HgTe devices); by the Center for Probing the Nanoscale, an NSF NSEC, supported under Grant No. PHY-0830228 (development of the SGM technique); and by the DARPA MESO Project under Grant No. N66001-11-1-4105 (MBE growth of the HgTe heterostructures). M. K. acknowledges financial support from the DAAD (Deutscher Akademischer Austausch-Dienst, German Academic Exchange Service).

[1] C. L. Kane and E. J. Mele, Quantum Spin Hall Effect in Graphene, Phys. Rev. Lett. 95, 226801 (2005).

[2] B. A. Bernevig and S.-C. Zhang, Quantum Spin Hall Effect, Phys. Rev. Lett. 96, 106802 (2006).

[3] C. Wu, B. A. Bernevig, and S.-C. Zhang, Helical Liquid and the Edge of Quantum Spin Hall Systems, Phys. Rev. Lett. 96, 106401 (2006).

[4] C. Xu and J. E. Moore, Stability of the Quantum Spin Hall Effect: Effects of Interactions, Disorder, and $\mathrm{Z}_{2}$ Topology, Phys. Rev. B 73, 045322 (2006).

[5] B. A. Bernevig, T. L. Hughes, and S.-C. Zhang, Quantum Spin Hall Effect and Topological Phase Transition in HgTe Quantum Wells, Science 314, 1757 (2006).

[6] C.-X. Liu, H.-J. Zhang, B. Yan, X.-L. Qi, T. Frauenheim, X. Dai, Z. Fang, and S.-C. Zhang, Oscillatory Crossover from Two-Dimensional to Three-Dimensional Topological Insulators, Phys. Rev. B 81, 041307(R) (2010).

[7] M. König, S. Wiedmann, C. Brüne, A. Roth, H. Buhmann, L. W. Molenkamp, X.-L. Qi, and S.-C. Zhang, Quantum Spin Hall Insulator State in HgTe Quantum Wells, Science 318, 766 (2007).

[8] A. Roth, C. Brüne, H. Buhmann, L. W. Molenkamp, J. Maciejko, X.-L. Qi, and S.-C. Zhang, Nonlocal Transport in the Quantum Spin Hall State, Science 325, 294 (2009).

[9] C. Brüne, A. Roth, H. Buhmann, E. M. Hankiewicz, L. W. Molenkamp, J. Maciejko, X.-L. Qi, and S.-C. Zhang, Spin Polarization of the Quantum Spin Hall Edge States, Nat. Phys. 8, 485 (2012).
[10] M. König, H. Buhmann, L. W. Molenkamp, T. Hughes, C.-X. Liu, X.-L. Qi, and S.-C. Zhang, The Quantum Spin Hall Effect: Theory and Experiment, J. Phys. Soc. Jpn. 77, 031007 (2008).

[11] J. Maciejko, C.-X. Liu, Y. Oreg, X.-L. Qi, C. Wu, and S.-C. Zhang, Kondo Effect in the Helical Edge Liquid of the Quantum Spin Hall State, Phys. Rev. Lett. 102, 256803 (2009).

[12] T. L. Schmidt, S. Rachel, F. von Oppen, and L. I. Glazman, Inelastic Electron Backscattering in a Generic Helical Edge Channel, Phys. Rev. Lett. 108, 156402 (2012).

[13] A. Ström, H. Johannesson, and G. I. Japaridze, Edge Dynamics in a Quantum Spin Hall State: Effects from Rashba Spin-Orbit Interaction, Phys. Rev. Lett. 104, 256804 (2010).

[14] J. I. Väyrynen and T. Ojanen, Electrical Manipulation and Measurement of Spin Properties of Quantum Spin Hall Edge States, Phys. Rev. Lett. 106, 076803 (2011).

[15] J. C. Budich, F. Dolcini, P. Recher, and B. Trauzettel, Phonon-Induced Backscattering in Helical Edge States, Phys. Rev. Lett. 108, 086602 (2012).

[16] M. A. Eriksson, R. G. Beck, M. Topinka, J. A. Katine, R. M. Westervelt, K. L. Campman, and A.C. Gossard, Cryogenic Scanning Probe Characterization of Semiconductor Nanostructures, Appl. Phys. Lett. 69, 671 (1996).

[17] M. A. Topinka, B. J. LeRoy, S. E. J. Shaw, E. J. Heller, R. M. Westervelt, K. D. Maranowski, and A. C. Gossard, Imaging Coherent Electron Flow from a Quantum Point Contact, Science 289, 2323 (2000).

[18] A. Pioda, S. Kičin, T. Ihn, M. Sigrist, A. Fuhrer, K. Ensslin, A. Weichselbaum, S. E. Ulloa, M. Reinwald, and W. Wegscheider, Spatially Resolved Manipulation of Single Electrons in Quantum Dots Using a Scanned Probe, Phys. Rev. Lett. 93, 216801 (2004).

[19] S. Schnez, J. Güttinger, M. Huefner, C. Stampfer, K. Ensslin, and T. Ihn, Imaging Localized States in Graphene Nanostructures, Phys. Rev. B 82, 165445 (2010).

[20] M. Huefner, B. Kueng, S. Schnez, K. Ensslin, T. Ihn, M. Reinwald, and W. Wegscheider, Spatial Mapping and Manipulation of Two Tunnel-Coupled Quantum Dots, Phys. Rev. B 83, 235326 (2011).

[21] N. Aoki, R. Brunner, A. M. Burke, R. Akis, R. Meisels, D. K. Ferry, and Y. Ochiai, Direct Imaging of Electron States in Open Quantum Dots, Phys. Rev. Lett. 108, 136804 (2012).

[22] S. Schnez, C. Rössler, T. Ihn, K. Ensslin, C. Reichl, and W. Wegscheider, Imaging the Lateral Shift of a Quantum Point Contact Using Scanning Gate Microscopy, Phys. Rev. B 84, 195322 (2011).

[23] M. A. Topinka, B. J. LeRoy, R. M. Westervelt, S. E. J. Shaw, R. Fleischmann, E. J. Heller, K. D. Maranowski, and A.C. Gossard, Coherent Branched Flow in a TwoDimensional Electron Gas, Nature (London) 410, 183 (2001).

[24] M. P. Jura, M. A. Topinka, L. Urban, A. Yazdani, H. Shtrikman, L. N. Pfeiffer, K.W. West, and D. Goldhaber-Gordon, Unexpected Features of Branched Flow through High-Mobility Two-Dimensional Electron Gases, Nat. Phys. 3, 841 (2007). 
[25] M. P. Jura, M. A. Topinka, M. Grobis, L. N. Pfeiffer, K. W. West, and D. Goldhaber-Gordon, Electron Interferometer Formed with a Scanning Probe Tip and Quantum Point Contact, Phys. Rev. B 80, 041303(R) (2009).

[26] M. P. Jura, M. Grobis, M. A. Topinka, L. N. Pfeiffer, K. W. West, and D. Goldhaber-Gordon, Spatially Probed Electron-Electron Scattering in a Two-Dimensional Electron Gas, Phys. Rev. B 82, 155328 (2010).

[27] A. Bachtold, M. S. Fuhrer, S. Plyasunov, M. Forero, E. H. Anderson, A. Zettl, and P. L. McEuen, Scanned Probe Microscopy of Electronic Transport in Carbon Nanotubes, Phys. Rev. Lett. 84, 6082 (2000).

[28] M. T. Woodside and P.L. McEuen, Scanned Probe Imaging of Single-Electron Charge States in Nanotube Quantum Dots, Science 296, 1098 (2002).

[29] A. C. Bleszynski-Jayich, L. E. Fröberg, M. T. Björk, H. J. Trodahl, L. Samuelson, and R. M. Westervelt, Imaging a One-Electron InAs Quantum Dot in an InAs/InP Nanowire, Phys. Rev. B 77, 245327 (2008).

[30] N. Harjee, A. G. F. Garcia, M. König, J. C. Doll, D. Goldhaber-Gordon, and B.L. Pruitt, in IEEE 23rd International Conference on Micro Electro Mechanical Systems (MEMS), Hong Kong, 2010 (IEEE, Piscataway, NJ, 2010), p. 344-347.

[31] B. Zhou, H.-Z. Lu, R.-L. Chu, S.-Q. Shen, and Q. Niu, Finite Size Effects on Helical Edge States in a Quantum Spin-Hall System, Phys. Rev. Lett. 101, 246807 (2008).

[32] M. Wada, S. Murakami, F. Freimuth, and G. Bihlmayer, Localized Edge States in Two-Dimensional Topological Insulators: Ultrathin Bi Films, Phys. Rev. B 83, 121310 (R) (2011).

[33] C.-Y. Hou, E.-A. Kim, and C. Chamon, Corner Junction as a Probe of Helical Edge States, Phys. Rev. Lett. 102, 076602 (2009).

[34] A. Ström and H. Johannesson, Tunneling between Edge States in a Quantum Spin Hall System, Phys. Rev. Lett. 102, 096806 (2009).
[35] T. L. Schmidt, Current Correlations in Quantum Spin Hall Insulators, Phys. Rev. Lett. 107, 096602 (2011).

[36] See Supplemental Material at http://link.aps.org/ supplemental/10.1103/PhysRevX.3.021003 for detailed information.

[37] M. Baenninger, M. König, A. G. F. Garcia, M. Mühlbauer, C. Ames, P. Leubner, C. Brüne, H. Buhmann, L. W. Molenkamp, and D. Goldhaber-Gordon, Fabrication of Samples for Scanning Probe Experiments on Quantum Spin Hall Effect in HgTe Quantum Wells, J. Appl. Phys. 112, 103713 (2012).

[38] X.-L. Qi, T.L. Hughes, and S.-C. Zhang, Fractional Charge and Quantized Current in the Quantum Spin Hall State, Nat. Phys. 4, 273 (2008).

[39] C. Timm, Transport through a Quantum Spin Hall Quantum Dot, arXiv:1111.2245.

[40] R. Ilan, J. Cayssol, J.H. Bardarson, and J.E. Moore, Nonequilibrium Transport through a Gate-Controlled Barrier on the Quantum Spin Hall Edge, Phys. Rev. Lett. 109, 216602 (2012).

[41] K. C. Nowack, E. M. Spanton, M. Baenninger, M. König, J. R. Kirtley, B. Kalisky, C. Ames, P. Leubner, C. Brüne, H. Buhmann, L.W. Molenkamp, D. GoldhaberGordon, and K. A. Moler, Imaging Currents in HgTe Quantum Wells in the Quantum Spin Hall Regime, arXiv:1212.2203.

[42] I. Knez, R.-R. Du, and G. Sullivan, Evidence for Helical Edge Modes in Inverted InAs/GaSb Quantum Wells, Phys. Rev. Lett. 107, 136603 (2011).

[43] C. Brüne, A. Roth, E. G. Novik, M. König, H. Buhmann, E. M. Hankiewicz, W. Hanke, J. Sinova, and L.W. Molenkamp, Evidence for the Ballistic Intrinsic Spin Hall Effect in HgTe Nanostructures, Nat. Phys. 6, 448 (2010).

[44] Not to be confused with ballistic backscattering of electrons by a tip more distant from a QPC, as shown in Ref. [17]. 